\title{
Non-destructive Photosynthetic Pigments Prediction using Multispectral Imagery and 2D-CNN
}

\author{
KESTRILIA REGA PRILIANTI1,2, SYAIFUL ANAM ${ }^{2}$, TATAS HARDO PANINTINGJATI \\ BROTOSUDARMO ${ }^{3}$, AGUS SURYANTO ${ }^{2}$ \\ 1 Department of Informatics Engineering, Universitas Ma Chung, Malang, 65151, Indonesia, (e-mail: kestrilia.rega@machung.ac.id) \\ 2 Department of Mathematics, Universitas Brawijaya, Malang, 65145, Indonesia, (e-mail: syaiful@ub.ac.id, suryanto@ub.ac.id) \\ 3 Ma Chung Research Center for Photosynthetic Pigments, Villa Puncak Tidar N-01, Malang, 65151, Indonesia, \\ (e-mail: tatas.brotosudarmo@machung.ac.id) \\ Corresponding author: Kestrilia Rega Prilianti (e-mail: kestrilia.rega@machung.ac.id).
}

\begin{abstract}
Rapid assessment of plant photosynthetic pigments content is an essential issue in precise management farming. Such an assessment can represent the status of plants in their stages of growth. We have developed a new 2 Dimensional-Convolutional Neural Network (2D-CNN) architecture, the P3MNet. This architecture simultaneously predicts the content of 3 main photosynthetic pigments of a plant leaf in a nondestructive and real-time manner using multispectral images. Those pigments are chlorophyll, carotenoid, and anthocyanin. By illuminating with visible light, the reflectance of individual plant leaf at 10 different wavelengths $-350,400,450,500,550,600,650,700,750$, and $800 \mathrm{~nm}$ - was captured in a form of 10 digital images. It was then used as the 2D-CNN input. Here, our result suggested that P3MNet outperformed AlexNet and VGG-9. After undergoing a training process using Adadelta optimization method for 1000 epochs, P3MNet has achieved superior MAE (Mean Absolute Error) in the average of $0.000778 \pm 0.0001$ for training and $0.000817 \pm 0.0007$ for validation (data range $0-1$ ).
\end{abstract}

:KEYWORDS Convolutional Neural Network; Multispectral Image; Non-Destructive; Photosynthetic Pigments.

\section{INTRODUCTION}

Dhotosynthetic pigments in plants have essential roles in the process of plant growth. They harvest solar light energy and use it for photosynthesis. Therefore, any changes in their contents can represent various conditions such as nutritional status, senescence, responses to environmental changes [1] as well as pests and diseases attack [2]. Hence, the development of rapid analysis methods of photosynthetic pigment contents is an important topic in agricultural research.

In recent years, advances in computer technology and electronics have led agricultural research to the development of pigment analysis methods in a non-destructive manner [3]. Non-destructive methods allow the quantification of pigment contents to be done in-situ and in real-time, and, thus, a rapid analysis can be easily performed. Gitelson et al. [4-6] have developed several non-destructive methods based on spectral reflectance from spectrophotometer-based measurements to predict the content of three main photosynthetic pigments (chlorophyll, carotenoid, anthocyanin) in plant leaves. Although the predictions are claimed to be quite good, spectrometer-based measurements are generally costly. Other researchers then developed an image-based measurement as an alternative [7]. These methods have been proven to be efficient, accurate, and easy to implement. In general, the RGB format is used [8-10]. However, in the case of simultaneous measurement of pigment contents, the RGB format is not enough. This refers to the theory that each type of pigment has a unique light reflectance behavior at certain wavelengths. The RGB format is mostly produced by camera 
sensors that have been filtered only to be able to capture reflections in the red $(620-750 \mathrm{~nm})$, green $(495-570 \mathrm{~nm})$ and blue (450-495) ranges. Therefore, using the RGB format as the raw data will eliminate a lot of important information that can be obtained from other wavelengths. For example, the reflectance in the near-infrared range $(750-800 \mathrm{~nm})$, which carries information about the diversity of leaf structure and thickness and as a correction for the calculation of pigment content in leaves containing both chlorophyll and anthocyanin [11]. Hence, in this study, we propose the use of multispectral digital images consisting of 10-channels. With reference to the positive results in our preliminary study using different species and without the wet chemical procedure [12], we hypothesized that, the prediction of photosynthetic pigments content in plant leaves would be approaching the results given by the spectrophotometerbased measurements and certainly better than those of 3channel (RGB) images. In 10-channel images, the quantity of light reflectance that represents the content of the photosynthetic pigments becomes more thorough and guides the system to produce better predictions.

To provide real-time analysis, an image-based measurement needs to be equipped with a machine learning method that is used to perform either classification [13] or regression [14] tasks on the image. In this study, we used the 2D-Convolutional Neural Network (2D-CNN) to perform a regression task on our pigments prediction system. This method is a variant of the Artificial Neural Network (ANN) that is now popularly used to handle input in the form of digital images. By applying the convolution method, the use of hyperparameter on $2 \mathrm{D}-\mathrm{CNN}$ is more efficient so that the learning process can be done quickly even with a large dimension of inputs [15] and minimize dependencies on human knowledge in determining the main features of the input images [16]. In conventional ANN, manual determination of the input main features is the most critical task to ensure the model performance. But on 2D-CNN, the features that represent the input image are generated automatically and continuously adjusted throughout the learning process without human bias. Moreover, 2D-CNN can also extract information from a digital image that consists of many color components (multispectral) simultaneously. In pigment analysis, each color component in a multispectral image carries unique information. Therefore we need a tool that can perform simultaneous and automatic extraction of all color components, such as the convolution method. This makes 2D-CNN superior to conventional ANN, especially in handling multispectral digital images.

\section{MATERIAL AND METHODS}

\section{A. THE SAMPLE}

Four species of Indonesian herbal plants were used in the experiment: Syzygium oleana, Piper betle, Jasminum sp. and Graptophyllum pictum. Each species was chosen carefully to be able to represent the diversity of the chlorophyll, carotenoid, and anthocyanin [17]. These pigments have a major role in the photosynthesis process. Also, compared to other pigments, they are more easily observed visually. $S$. oleana contained high concentrations of carotenoid and anthocyanin, while $G$. pictum contained high concentrations of anthocyanin and chlorophyll. Jasminum sp contained high concentrations of chlorophyll and carotenoid. If there is a predominance of certain pigments in these three varieties, then $P$. betle is not the case. Hence, $P$. betle played a role to complement the other diversity that could not be obtained from the other three varieties. Part of the plants sampled in this study were leaves. The sample diversity of color, age, and position from the terminal bud was among our consideration during the sample preparation. A total of 212 fresh leaves were taken from several regions in Malang, East Java, Indonesia.

\section{B. DATA ACQUISITION}

Two data acquisition processes were applied to each leaf on the same day. The first process was the acquisition of its multispectral image and the second process was the acquisition of photosynthetic pigment content by wet chemical methods.

\section{B.1. MULTISPECTRAL IMAGE ACQUISITION}

Fig 1. depicts the devices arrangement. The leaf sample was placed in a tray with special clips. A bandpass filter with 10 channels - 350, 400, 450, 500, 550, 600, 650, 700, 750, and $800 \mathrm{~nm}$ - was placed between the CCD camera and the leaf samples. Tungsten halogen was used as the light source since it provides a wide range of electromagnetic wavelengths from 360 up to $2400 \mathrm{~nm}$.

An image of the leaf was then taken with a CCD camera (Pcopixelfly 14 bit). A reflectance spectrophotometer (Ocean Optic USB- 4000) was also used to capture the reflectance spectrum of each leaf sample. The spectra of the samples were used for calibration and validation. Fig. 2 depicts an example of the multispectral images taken from a leaf sample. Dark-colored images indicate the lack of reflectance of leaf samples at certain wavelengths and vice versa. The images labeled 350, 400, 450 and $500 \mathrm{~nm}$ appear quite dark. This visualization shows that the leaf sample is very little or even does not reflect light at these ranges.

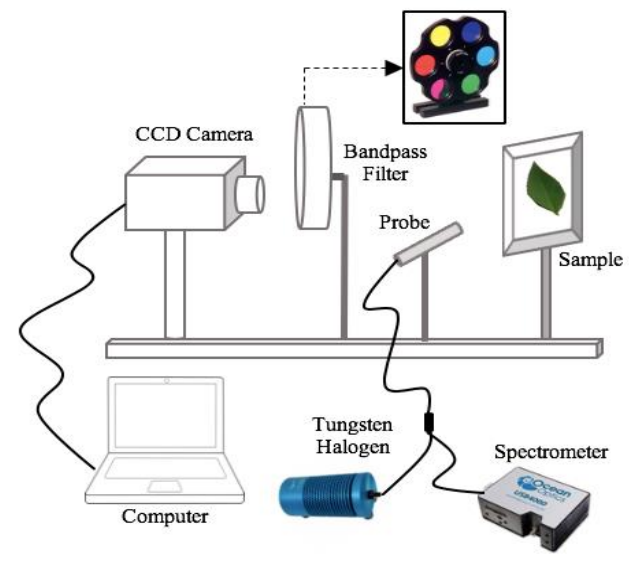


Figure 1. The devices arrangement for multispectral image acquisition

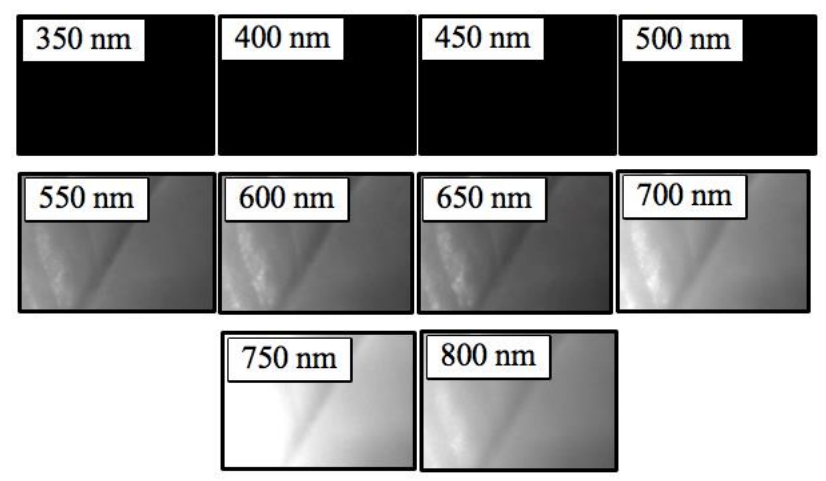

Figure 2. The example of a leaf multispectral image

\section{B. PIGMENT CONTENTS MEASUREMENT}

Each leaf was divided into 2 parts, one for the chlorophyll and carotenoid measurement and the other for the anthocyanin measurement. Using a mortar and pestle, they were mashed into small pieces. As much as 0.05 grams were then withdrew and put into different tubes. The pigments were then extracted by adding the $\mathrm{CaCO}_{3}$ and sodium ascorbate powder with $1.5 \mathrm{~mL}$ of solvent into the tubes. Solvent for chlorophyll and carotenoid were $100 \%$ acetone, whereas a mixture of methanol, concentrated hydrochloric acid and distilled water was used for anthocyanin. Homogenization of the mixture was then carried out for 1 minute using a vortex. For the next 1 minute, the tubes were immersed in the ice cubes. The homogenization process was repeated 3 times before all tubes were centrifuged at 14000 rpm for two minutes and cooled again with the ice cubes. The absorbance measurement was done using double-beam UV/VIS scanning spectrophotometer (Shimadzu Corp., Kyoto, Japan) and the conversion of the absorbance values into pigment contents $(\mu \mathrm{g} / \mathrm{g})$ was done using Lichenthaler [18] and Sims and Gammons [19] formula. At the end, for each leaf, 3 sets of data were acquired, i.e., the chlorophyll content, the carotenoid content and the anthocyanin content.

\section{DESIGN OF THE 2D-CNN ARCHITECTURES}

We have developed 3 of our original 2D-CNN architectures and compared them with 2 well-known architectures, i.e., AlexNet and VGG-9. Our original architecture is named P3MNet, which stands for Plant Pigment Prediction Multispectral Network. The input was a 10 channels digital image of a plant leaf while the output was the prediction of chlorophyll, carotenoid, and anthocyanin contents. Table 1 shows the details of the five architectures used in the experiment. These five architectures are carefully arranged to represent the level of network complexity. The architectures on the left column side are less complex compared to those on the right column side. The architecture with the highest complexity is VGG-9 and the lowest is P3MNet_1. Prior to the experiment, we modified AlexNet and VGG-9, which are generally used for classification tasks. In this study, AlexNet and VGG-9 are modified for regression tasks.

We used a total of 212 10-channel multispectral images for the training process. Each architecture was trained using 7 gradient descent-based optimization methods: Stochastic Gradient Descent (SGD) [20], Adaptive Gradient (Adagrad) [21], Adaptive Delta (Adadelta) [22], Root Mean Square Propagation (RMSProp) [23], Adaptive Momentum (Adam) [24], Adaptive Max Pooling (Adamax) [24], and Nesterov Adaptive Momentum (Nadam) [25]. We carefully select the optimization method that is most suitable for each architecture. Thus, the results reported in this article are the most accurate results among all possible outcomes. We have also tried to apply the hessian-based optimization method. However, the training time is almost two times longer than the training time for the gradient-based method. This is due to heavy computations to create the hessian matrix of the loss and without the concept of batch, the training process is forced to receive the training set at once in one epoch. Moreover, we found that the MAE of the hessian-based method is relatively equal to the MAE of the gradient-based method. Therefore, we did not continue the experiment with the hessian-based optimization method.

Table 1. The 2D-CNN architectures that were experimented on

\begin{tabular}{|c|c|c|c|c|c|c|}
\hline \multirow{2}{*}{\multicolumn{2}{|c|}{$\begin{array}{l}\text { Layer } \\
\text { Input Image } \\
\text { Size }\end{array}$}} & P3MNet_1 & P3MNet_2 & P3MNet_3 & AlexNet & VGG-9 \\
\hline & & $34 \times 34 \times 10$ & $34 \times 34 \times 10$ & $120 \times 120 \times 10$ & $120 \times 120 \times 10$ & $120 \times 120 \times 10$ \\
\hline \multirow{7}{*}{ 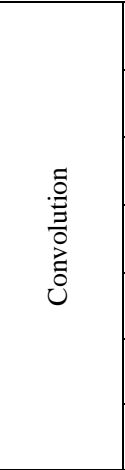 } & 1 & $\begin{array}{l}32 \text { kernels in size } 3 \times 3 \\
\text { with max pooling }\end{array}$ & 16 kernels in size $3 \times 3$ & $\begin{array}{l}60 \text { kernels in size } 5 \times 5 \\
\text { with max pooling }\end{array}$ & $\begin{array}{l}96 \text { kernels in size } 5 \times 5 \\
\text { with max pooling }\end{array}$ & 64 kernels in size $5 \times 5$ \\
\hline & 2 & $\begin{array}{l}32 \text { kernels in size } 3 \times 3 \\
\text { with max pooling }\end{array}$ & $\begin{array}{l}16 \text { kernels in size } 3 \times 3 \\
\text { with max pooling }\end{array}$ & $\begin{array}{l}90 \text { kernels in size } 3 \times 3 \\
\text { with max pooling }\end{array}$ & $\begin{array}{l}256 \text { kernels in size } \\
3 \times 3 \text { with max pooling }\end{array}$ & $\begin{array}{l}64 \text { kernels in size } 3 \times 3 \\
\text { with max pooling }\end{array}$ \\
\hline & 3 & - & $\begin{array}{l}16 \text { kernels in size } 3 \times 3 \\
\text { with max pooling }\end{array}$ & $\begin{array}{l}120 \text { kernels in size } \\
3 \times 3 \text { with max pooling }\end{array}$ & $\begin{array}{l}384 \text { kernels in size } \\
3 \times 3\end{array}$ & $\begin{array}{l}128 \text { kernels in size } \\
3 \times 3\end{array}$ \\
\hline & 4 & - & - & - & $\begin{array}{l}256 \text { kernels in size } \\
3 \times 3 \text { with max pooling }\end{array}$ & $\begin{array}{l}128 \text { kernels in size } \\
3 \times 3 \text { with max pooling }\end{array}$ \\
\hline & 5 & - & - & - & - & $\begin{array}{l}256 \text { kernels in size } \\
3 \times 3\end{array}$ \\
\hline & 6 & - & - & - & - & $\begin{array}{l}256 \text { kernels in size } \\
3 \times 3\end{array}$ \\
\hline & 7 & - & - & - & - & $\begin{array}{l}256 \text { kernels in size } \\
3 \times 3 \text { with max pooling }\end{array}$ \\
\hline \multirow{2}{*}{ I $\Xi \gtrsim$} & 1 & 90 nodes, ReLu & 90 nodes, ReLu & 100 nodes, ReLu & 4096 nodes, ReLu & 4096 nodes, ReLu \\
\hline & 2 & - & - & 100 nodes, ReLu & 4096 nodes, ReLu & 4096 nodes, ReLu \\
\hline
\end{tabular}


The ReLu activation function was used in the convolutional and fully connected layer. In the output nodes we used LeakyReLu [26] to avoid a dead ReLu. The LeakyReLu activation function is:

$$
\begin{gathered}
h^{(i)}=\max \left(w^{(i) T} x, 0\right), \\
w^{(i) T} x=\left\{\begin{array}{c}
w^{(i) T} x, w^{(i) T} x>0 \\
0.1 w^{(i) T}, \text { else }
\end{array}\right.
\end{gathered}
$$

where $w^{(i)}$ is the weight vector for the $i$-th hidden unit and $x$ is the input. Due to differences in the amount of pigment content, - chlorophyll ranges in a hundred while anthocyanin and carotenoid range in the tens - we normalize the target data into a $0-1$ range. The normalization was done using

$$
Z=\frac{x-\min (x)}{[\max (x)-\min (x)]}
$$

where $z$ is the normalized data and $x$ is the raw data. The normalized data will ensure that each neuron (kernel) in the 2D-CNN has an equal chance to learn the variation of each pigment content. In addition, normalization will also speed up the calculation process for the hyperparameter updates. Mean Absolute Error (MAE) was used as the performance indicator,

$$
\text { MAE }=\frac{1}{n} \sum_{i=1}^{n}\left|y_{i}-\widehat{y}_{i}\right|,
$$

where $y_{i}$ is the actual pigment content, $\hat{y}_{i}$ is the predicted pigment content and $n$ is the sample size. The optimization methods task is to minimize the MAE.

\section{DATA AUGMENTATION}

One of the issues that we face in this study is related to the time and cost of wet chemical procedures. This precludes the production of large data, which is generally a requirement of CNNs. However, some previous studies have claimed that CNNs can still show good performance with small data [2728]. We have found that augmentation techniques can be applied to overcome underfitting and overfitting problems that often occur in the case of small data learning processes [29]. In this study, we applied a spatial-based augmentation. We created various variations in the position of the leaf image using rotation. Fig. 3 compares the P3MNet_3's MAE with and without the application of augmentation techniques. It can be seen that without augmentation, the fluctuations in MAE values in both the training and validation processes are enormous. This shows the instability of the predictions. With augmentation, the fluctuations in the MAE considerably decrease. In addition to the fluctuation problem, we also faced the problem of underfitting. It can be found in the MAE value at the end of the training epoch. Without augmentation, the MAE is much greater $( \pm 0.0203)$ when compared to the MAE with augmentation $( \pm 0.000778)$.

\section{E. EXPERIMENTAL SETUP}

Python 2 was used to develop the 2D-CNN architectures. The TensorFlow backend along with the Keras API and GPU support was used to enable fast calculations. The experiment was run with the support of Google Colaboratory facilities and on a personal computer with the macOS Sierra operating system, Intel Core i5 1.6 GHz processor, and $8 \mathrm{~GB}$ of DDR3 RAM.

\section{RESULT AND DISCUSSION}

\section{A. Pigment Contents and Composition}

The wet chemical procedure produces data of pigment contents from each leaf samples as summarized in Table 2. $S$. oleana and $G$. pictum leaves contain a higher concentration of anthocyanin than the other two species. Jasminum sp. contains more chlorophyll and carotenoid, whereas P. betle did not appear to have a significant dominating pigment. Therefore, it fills in the other diversity of pigments composition which has not been fulfilled by the other three species. Since variations in the samples are the most important success factor in the 2D-CNN learning process, it is important to ensure that the required variations are sufficiently represented by the leaf samples.

Table 2. Statistical summary of the pigment content measured by wet chemical procedure

\begin{tabular}{|c|c|c|}
\hline Species & Pigment Name & Pigment Content $(\mu \mathrm{g} / \mathrm{g}) *$ \\
\hline \multirow{4}{*}{ S. oleana } & Chlorophyll & $124.21 \pm 147.95$ \\
\cline { 2 - 3 } & Carotenoid & $31.97 \pm 32.84$ \\
\cline { 2 - 3 } & Anthocyanin & $59.45 \pm 73.33$ \\
\hline \multirow{4}{*}{ P. betle } & Chlorophyll & $30.48 \pm 37.23$ \\
\cline { 2 - 3 } & Carotenoid & $8.94 \pm 9.92$ \\
\cline { 2 - 3 } & Anthocyanin & $4.58 \pm 6.61$ \\
\hline \multirow{4}{*}{ Jasminum sp. } & Chlorophyll & $306.57 \pm 171.83$ \\
\cline { 2 - 3 } & Carotenoid & $44.24 \pm 39.29$ \\
\cline { 2 - 3 } & Anthocyanin & 0 \\
\hline \multirow{3}{*}{ G. pictum } & Chlorophyll & $169.75 \pm 161.54$ \\
\cline { 2 - 3 } & Carotenoid & $26.58 \pm 26.08$ \\
\cline { 2 - 3 } & Anthocyanin & $47.32 \pm 67.32$ \\
\hline
\end{tabular}

* Pigment content relative to the dry weight of the leaf material 


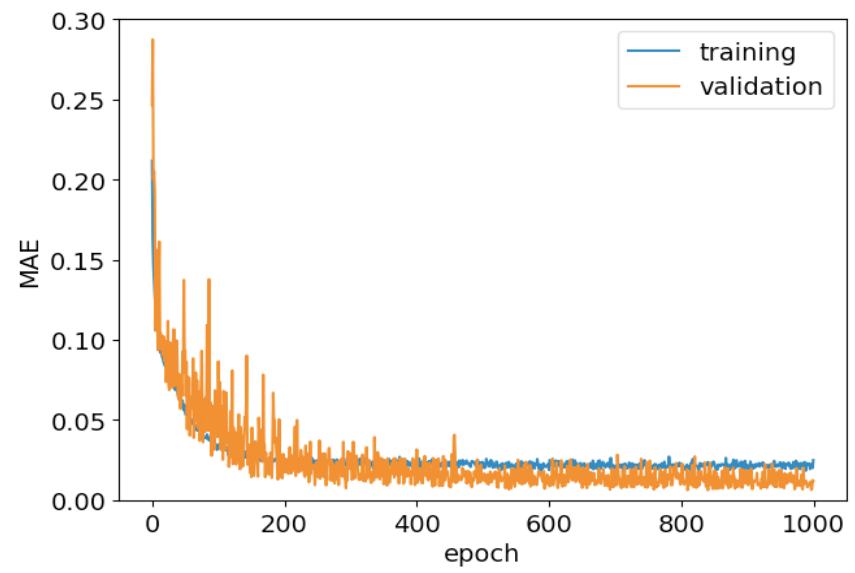

(a)

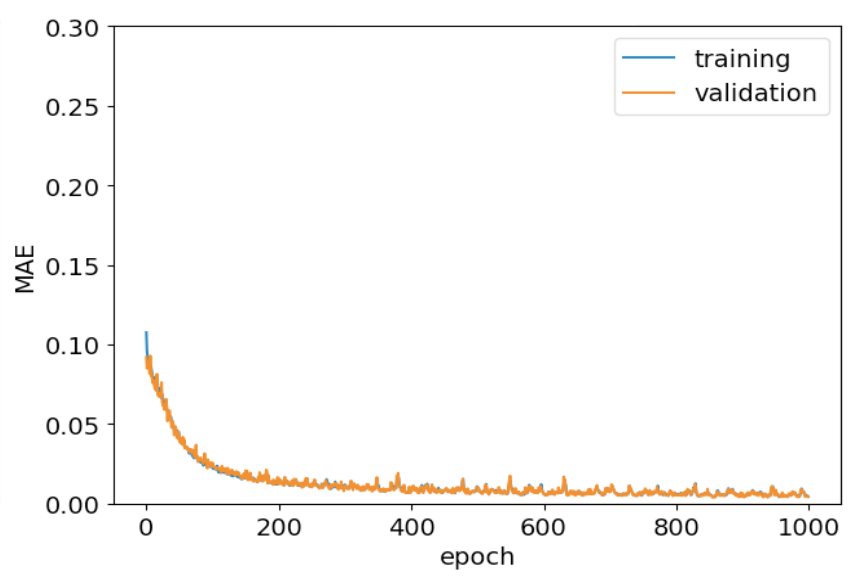

(b)

Figure 3. The MAE alteration before and after the application of augmentation process, (a) Before augmentation, (b) After augmentation

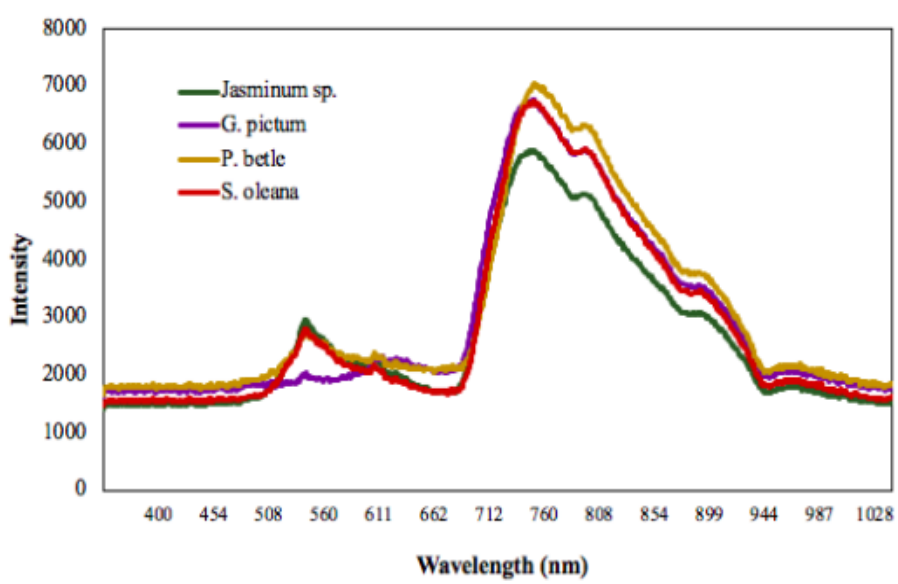

\begin{tabular}{|c|r|r|r|r|}
\hline \multirow{2}{*}{ Species } & Chlorophyll & Carotenoid & Anthocyanin & Water Content \\
\cline { 2 - 5 } & $(\mu \mathrm{g} / \mathrm{g})$ & $(\mu \mathrm{g} / \mathrm{g})$ & $(\mu \mathrm{g} / \mathrm{g})$ & $(\%)$ \\
\hline Jasminum sp. & 397.55 & 28.46 & 0.00 & 62.48 \\
\hline G. pictum & 359.08 & 61.30 & 299.76 & 61.27 \\
\hline P. betle & 94.66 & 19.10 & 7.34 & 73.15 \\
\hline S. oleana & 240.04 & 67.17 & 12.30 & 42.10 \\
\hline
\end{tabular}

Figure 4. Spectral reflectance example of four individual leaf samples from four different species and its pigments and water content

From Table 2, we can find out that the variation of pigment content for each species varies widely, it can be seen from the large standard deviation values. Thus, we have confirmed that sufficient variation had been successfully met in this study. The example of leaf reflectance behavior to a visible light at various wavelengths can be seen in Fig. 4. It shows a comparison of the reflectance spectra of 4 individual leaves from 4 different species measured by a spectrometer. In general, the chlorophyll, carotenoid, and anthocyanin content in a plant leaf can be observed from the combination of its reflectance on three spectral ranges, i.e., (540-560 nm, $710-720 \mathrm{~nm}$, and 770-800 nm) [4]. In Fig. 4, the difference is quite clear at those spectral ranges. The difference in pattern is created due to the simultaneous influence of the photosynthetic pigments contained in each leaf.

However, leaf thickness and water content also contribute to pattern diversity. Therefore, reflectance at other spectral ranges is also important to adjust the predicted pigment content to produce more accurate results. Hence, the complexity of the light reflectance behavior by a plant leaf is formed. Such complexity results in a high non-linear relationship between the reflectance pattern and the content of photosynthetic pigments. Therefore, in this study, 2DCNNs is used to represent such a difficult relationship.

\section{B. MODEL DEVELOPMENT AND VALIDATION}

Adadelta was the best optimization method to train AlexNet and VGG-9 [30]. Fig. 5 shows a comparison of the P3MNet_3's overall MAE trained with seven different optimization methods. It appears that Adadelta also gives the smallest MAE. The same results also apply to the other two P3MNet architectures. Therefore, all P3MNet architectures in this experiment were trained using the Adadelta optimization method. 


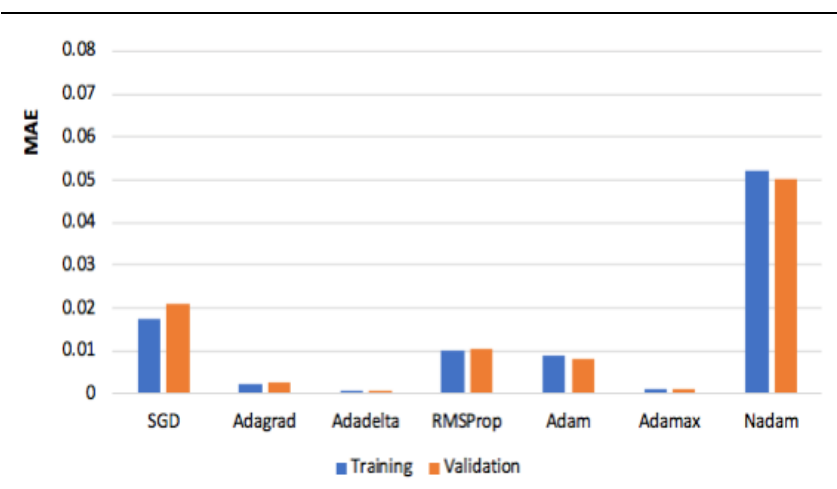

Figure 5. The MAE comparison of P3MNet_3 architecture trained with seven different optimization methods

The experimental results are summarized in Table 3. Each architecture underwent 10 learning processes to explore its behavior, each process went through 1000 epochs. Beyond the $1000^{\text {th }}$ epoch, there were no significant MAE changes. Of the five architectures that were experimented, P3MNet_3, AlexNet, and VGG-9 provides lower average of MAE. Thus, we found that the extreme addition of the number of kernels in the CNN convolution layers and nodes in the fully connected layers (see Table 1) did not significantly reduce the MAE. Compared to the P3MNet_V3's MAE, the MAE of AlexNet and VGG-9 seem slightly smaller. However, considering the number of parameters that must be managed, the MAE of AlexNet and VGG-9 are not quite encouraging. Fig. 6 shows the comparison of the file size of the trained model (in .h5 format) between the three CNN models. It appears that the trained P3MNet_V3 model is extremely more efficient in storage, which is not the case with AlexNet and VGG-9. Both of them require storage that is 7 times larger than P3MNet_V3. For the sake of developing portable devices, this condition is not favorable.

Table 3. The MAE comparison of the five architectures used in the experiment

\begin{tabular}{|l|l|c|}
\hline \multicolumn{2}{|c|}{ Architecture } & $\begin{array}{c}\text { MAE } \\
(\bar{x} \pm s)\end{array}$ \\
\hline \multirow{2}{*}{ P3MNet_1 } & Training & $0.0038 \pm 0.0002$ \\
\cline { 2 - 3 } & Validation & $0.0035 \pm 0.0003$ \\
\hline \multirow{2}{*}{ P3MNet_2 } & Training & $0.0047 \pm 0.0001$ \\
\cline { 2 - 3 } & Validation & $0.0035 \pm 0.0002$ \\
\hline \multirow{2}{*}{ P3MNet_3 } & Training & $0.000778 \pm 0.0005$ \\
\cline { 2 - 3 } & Validation & $0.000817 \pm 0.0007$ \\
\hline \multirow{2}{*}{ ALexNet } & Training & $0.000633 \pm 0.0034$ \\
\cline { 2 - 3 } & Validation & $0.000598 \pm 0.0044$ \\
\hline \multirow{2}{*}{ VGG-9 } & Training & $0.000592 \pm 0.0041$ \\
\cline { 2 - 3 } & Validation & $0.000588 \pm 0.0040$ \\
\hline
\end{tabular}

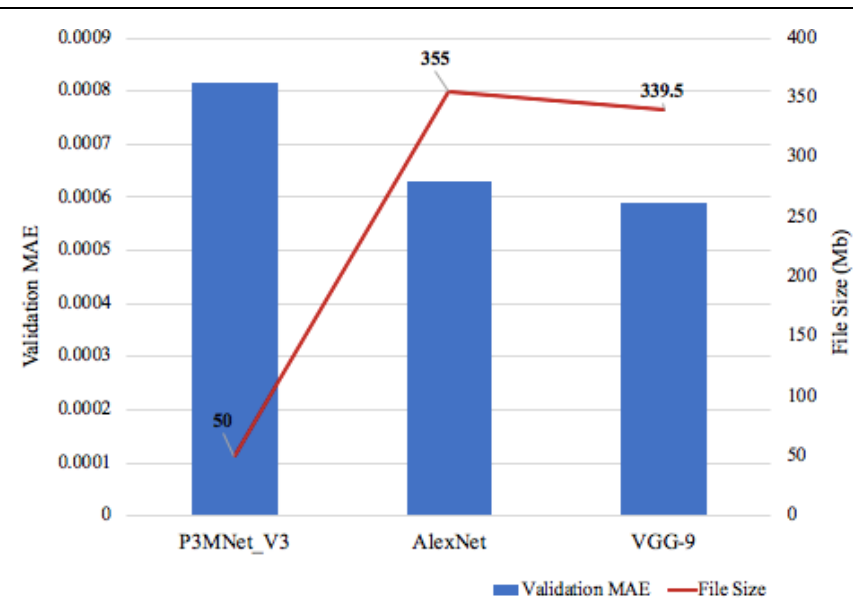

Figure 6. The MAE comparison of P3MNet_3, AlexNet and VGG-9 together with their trained models file size.

It can be concluded that increasing the number of convolution layers does not make predictions significantly better. This behavior is also in line with the theory that with the limited number of samples, the complexity of CNN cannot be too high [31]. Therefore, P3MNet_3 was chosen as the best model to represent the relationship between 10 channels of leaf image and their photosynthetic pigment contents.

Fig. 7 shows the performance of P3MNet_3 for each pigment. It appears that for the 3 pigments, the correlation between observed pigment content and estimated pigment content is very good; it is positive and the correlation coefficient (R) is close to one. This result is also superior when compared to the non-destructive technique developed by Gitelson et al. [32]. Using a spectrum measured with a spectrometer, Gitelson et al. have created indices that are claimed to be very good at predicting chlorophyll, carotenoid, and anthocyanin content of plant leaves nondestructively. The indices are (Chl) RI (Chlorophyll Reflectance Index), CRI (Carotenoid Reflectance Index), and ARI (Anthocyanin Reflectance Index). Each index gives a correlation value $(\mathrm{R})$ of $0.959,0.969$, and 0.967, respectively. This data show that the use of $2 \mathrm{D}-\mathrm{CNN}$ with a 10 channels digital image can replace the use of the reflectance index with the reflectance spectrum data (measured with a spectrometer). Both are proven to provide almost the same accuracy.

However, when examined in more detail, we found little differences in the MAE. Fig. 8 depicts a boxplot of the validation MAE for all three pigments. Anthocyanin seems to be more predictable. It can be seen from its MAE which is lower compared to those for the other two pigments. Furthermore, the fluctuation in MAE values for anthocyanin is also the lowest. It is an indicator that P3MNet_3 can provide better consistency in anthocyanin prediction. Of the three pigments, chlorophyll's MAE is the worst, its fluctuation also appears to be greater than anthocyanin. Meanwhile, the behavior of carotenoid's MAE tends to be more similar to that of chlorophyll This phenomenon occurs since the reflectance by chlorophyll (especially in the green 
spectral range) can be reduced in the presence of anthocyanin. This is usually found on leaves that contain both these pigments. Huang [33] also reported a similar phenomenon for the prediction of chlorophyll in sweet potatoes using spectral reflectance data and developed a new index to fix it. However, the method to deal with the same phenomenon for multispectral digital images remains unknown. This issue will be the focus of our next research project.
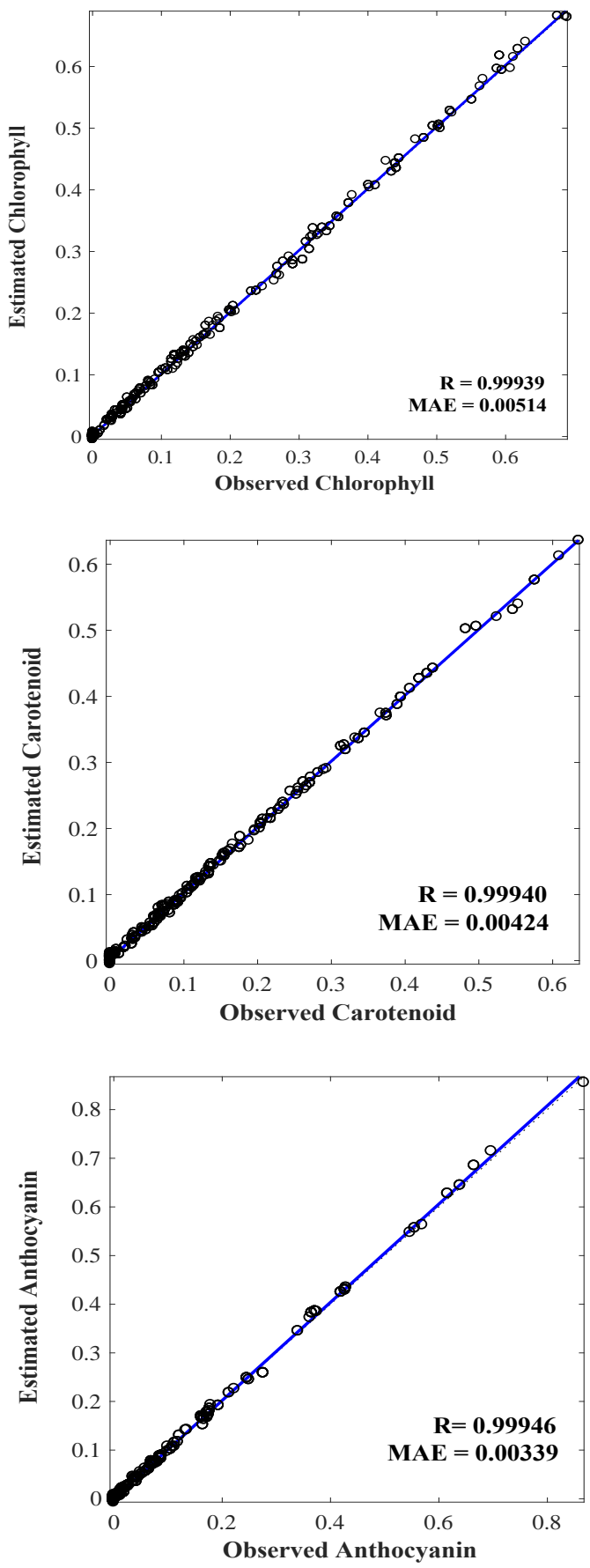

Figure 7. Performance of the P3MNet_3 for each pigment

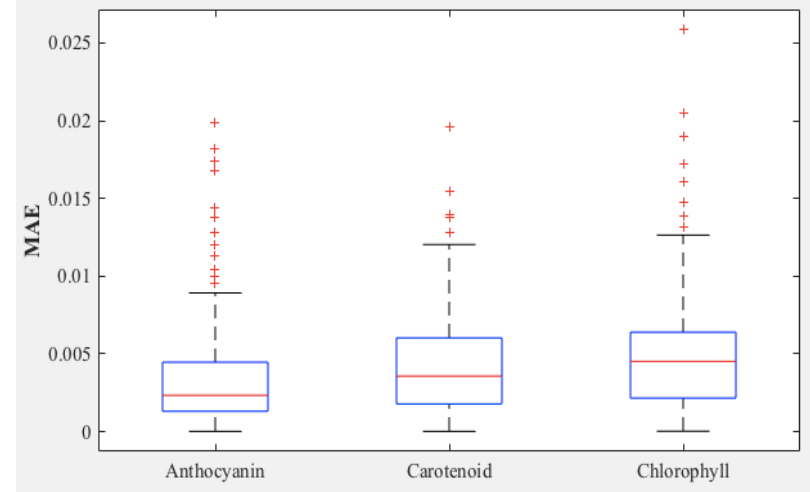

Figure 8. Boxplot of the validation MAE of P3MNet_3 for each pigment

\section{CONCLUSION}

In this study, we compared 5 2D-CNN architectures to predict the content of photosynthetic pigments in a plant leaf using its multispectral image. Of the five architectures, 3 of them are our original models named P3MNet. We found that the P3MNet_3 architecture is the best among all with training $\mathrm{MAE}=0.000778 \pm 0.0005$ and validation $\mathrm{MAE}=$ $0.000817 \pm 0.0007$ (data range $0-1$ ). In the input layer, there was a digital image of a plant leaf consisting of 10 channels. Each channel represents the reflectance of leaf sample to light at wavelengths $350,400,450,500,550,600,650,700$, 750 , and $800 \mathrm{~nm}$ respectively. The P3MNet_3 was trained using the Adadelta optimization method, run in 1000 epochs. Three main photosynthetic pigments, i.e., chlorophyll, carotenoid, and anthocyanin have been successfully predicted with high accuracy. However, there is a slight difference in the prediction performance for each of the three pigments. P3MNet_3 tends to give a smaller MAE value for anthocyanin compared to carotenoid and chlorophyll.

Based on these positive results, we have shown that a multispectral digital image along with 2D-CNN can be used as a good non-destructive tool for photosynthetic pigment content measurement in plant leaves. Its accuracy can compete with other non-destructive instruments that have been developed by previous researchers.

\section{References}

[1] R. Esteban, O. Barrutia, U. Artetxe, B. Fernández-Marín, A. Hernández, J.I. García-Plazaola, "Internal and external factors affecting photosynthetic pigment composition in plants: a metaanalytical approach," New Phytologist, vol. 206, issue 1, pp. 268-280, 2015. https://doi.org/10.1111/nph.13186.

[2] A. Jabeen, T.V. Kiran, D. Subrahmanyam, D.L. Lakshmi, G. Bhagyanarayana, D. Khrisnaveni, "Variations in chlorophyll and carotenoid contents in tungro infected rice plants," Journal of Research and Development, vol. 5, issue 1, pp. 2311-3278, 2017.

[3] H. Croft, J.M. Chen, Leaf Pigment Content, in: S. Liang, X. Xiong, J.J. Butler (Eds.), Comprehensive Remote Sensing, Elsevier Inc, 2018 , pp. 117-142. https://doi.org/10.1016/B978-0-12-409548-9.10547-0.

[4] A. Gitelson, Non-destructive Estimation of Foliar Pigment (Chlorophylls, Carotenoids and Anthocyanins) Contents: Espousing a Semi-Analytical Three-Band Model, in: Thenkabail, P.S., Lyon, J.G., Huete, A. (Eds.), Hyperspectral Remote Sensing of Vegetation. Taylor and Francis, 2011, pp. 141-162. 
[5] A. Gitelson, A. Solovchenko, "Generic algorithm for estimating foliar pigment content," Geophysical Research Letter, vol. 44, issue 18, pp. 9293-9298, 2017. https://doi.org/10.1002/2017GL074799.

[6] A. Gitelson, A. Solovchenko, "Non-invasive quantification of foliar pigments: Possibilities and limitations of reflectance and absorbancebased approaches," Journal of Photochemistry and Photobiology B: Biology, vol. 178, issue January, pp. 537-544, 2018. https://doi.org/10.1016/j.jphotobiol.2017.11.023.

[7] D. Inácio, R. Rieder, "Computer vision and artificial intelligence in precision agriculture for grain crops: a systematic review," Computer and Electronics in Agriculture, vol. 153, issue October, pp. 69-81, 2018. https://doi.org/10.1016/j.compag.2018.08.001.

[8] Y. Peng, Y. Wang, "Prediction of the chlorophyll content in pomegranate leaves based on digital image processing technology and stacked sparse autoencoder," International Journal of Food Properties, vol. 22, issue 1, pp. 1720-1732, 2019. https://doi.org/10.1080/10942912.2019.1675692.

[9] M. Perez-Patricio, J. Camas-Anzueto, A. Sánchez-Alegría, A. Aguilar-González, F. Gutiérrez-Miceli, "Optical method for estimating the chlorophyll contents in plant leaves," Sensors, vol. 18, issue 650, pp. 1-12, 2018. https://doi.org/10.3390/s18020650.

[10] M. Tanska, M Ambrosewicz-Walacik, K. Jankowski, D. Rotkiewicz, "Possibility use of digital image analysis for the estimation of the rapeseed maturity stage," International Journal of Food Properties, vol. 20, issue 3, pp. S2379-S2394, 2018. https://doi.org/10.1080/10942912.2017.1371188.

[11] A. Gitelson, M.N. Merzlyak, "Non-destructive assessment of chlorophyll, carotenoid and anthocyanin content in higher plant leaves: Principle and algorithms, in: S. Stamatiadis, J.M. Lynch, J.S. Schepers (Eds), Remote Sensing for Agriculture and the environment, Greece, Ella, 2004, pp. 78-94

[12] K.R. Prilianti, I.C. Onggara, M.A.S. Adiwibhawa, T.H.P. Brotosudarmo, "Multispectral imaging and convolutional neural network for photosynthetic pigments prediction," Proceedings of the $5^{\text {th }}$ International Conference on Electrical Engineering Computer Science and Informatics (EECSI), Malang, Indonesia, August 31 September 1, 2018, pp. 554-559. https://doi.org/10.1109/EECSI.2018.8752649.

[13] J.I. Arribas, G.V. Sanchez-Ferrero, G. Ruiz-Ruiz, and J. Gomez-Gil, "Leaf classification in sunflower crops by computer vision and neural networks," Computer and Electronics in Agriculture, vol. 78, issue 1, pp. 9-18, 2011. https://doi.org/10.1016/j.compag.2011.05.007.

[14] J.C. Pyo, H. Duan, S. Baek, M.S. Kim, T. Jeon, Y.S. Kwon, H. Lee, K.H. Cho, "A convolutional neural network regression for quantifying cyanobacteria using hyperspectral imagery," Remote Sensing of Environment, vol. 233, Issue November, pp. 111350, 2019. https://doi.org/10.1016/j.rse.2019.111350.

[15] J. Gu, Z. Wang, J. Kuen, L. Ma, A. Shahroudy, B. Shuai, T. Liu, X. Wang, L. Wang, G. Wang, J. Cai, T. Chen, "Recent advances in convolutional neural networks," Pattern Recognition, vol. 77, issue May, pp. 3018. https://doi.org/10.1016/j.patcog.2017.10.013.

[16] Y.L. Cun, Y. Bengio, and G. Hinton, "Deep learning," Nature, vol. 521, pp. 436-444, 2015. https://doi.org/10.1038/nature14539.

[17] K.R. Prilianti, S. Anam, A. Suryanto, "Deep chemometrics for nondestructive photosynthetic pigments prediction using leaf reflectance spectra," Information Processing in Agriculture, vol. 8, issue 1, pp. 194-204 2021. https://doi.org/10.1016/j.inpa.2020.02.001.

[18] H.K., Lichtenthaler, "Chlorophyll and carotenoids: pigments of photosynthetic biomembranes," Methods Enzymology, vol. 148, pp. 350-382, 1987. https://doi.org/10.1016/0076-6879(87)48036-1.

[19] D.A. Sims, J.A. Gamon, "Relationships between leaf pigment content and spectral reflectance across a wide range of species, leaf structures and developmental stages," Remote Sensing of Environment, vol. 81, issue 2-3, pp. 337-354, 2002. https://doi.org/10.1016/S00344257(02)00010-X.

[20] P. Toulis, T. Horel, E.M. Airoldi, "Stable Robbins-Monro approximations through stochastic proximal updates," arXiv preprint arXiv: $1510.00967 \mathrm{v} 3,2018$.

[21] J. Duchi, E. Hazan, Y. Singer, "Adaptive subgradient methods for online learning and stochastic optimization," Journal of Machine Learning Research, vol. 12, issue July, pp. 2121-2159, 2011

[22] M.D. Zeiler, "ADADELTA: an adaptive learning rate method," arXiv:1212. 5701, 2012

[23] G. Hinton, N. Srivastava, K. Swersky, "Overview of mini batch gradient descent," Computer Science Department, University of Toronto, 2015.

[24] D.P. Kingma, J.L. Ba, "Adam: a method for stochastic optimization," Proceedings of the International Conference on Learning Representations, San Diego, USA, May 7-9, 2015, pp. 1-15.

[25] T. Dozat, "Incorporating Nesterov momentum into adam," Proceedings of the International Conference on Learning Representation, San Juan, Puerto Rico, May 2-4, 2016, pp. 1-4.

[26] A.L. Maas, A.Y. Hannun, A.Y. Ng, "Rectifiers nonlinearities improve neural network acoustic models," Proceedings of $30^{\text {th }}$ International Conference on Machine Learning, Atlanta, USA, June 16-21, 2013, pp. 1-4.

[27] B. Gaonkar, D. Hovda, N.A. Martin, L. Macyszyn, "Deep learning in the small sample size setting: cascaded feedforward neural networks for medical image segmentation," Proceedings of the SPIE Medical Imaging, San Diego, USA, February 27 - March 3, 2016, pp. 1-8. https://doi.org/10.1117/12.2216555.

[28] R. Keshari, M. Vatsa, R. Singh, A. Noore, "Learning structure and strength of CNN filters for small sample size training," arXiv:1803.11405, 2018. https://doi.org/10.1109/CVPR.2018.00974.

[29] N.M.R, Aquino, M. Gutoski, L. Hattori, H.S. Lopes, "The effect of data augmentation on the performance of convolutional neural networks," Proceedings of the Brazilian Society of Computational Intelligence, Rio de Janeiro, Brazil, October, 2017, pp. 1-12. https://doi.org/10.21528/CBIC2017-51.

[30] K.R., Prilianti, T.H.P. Brotosudarmo, S. Anam, A. Suryanto, "Performance comparison of the convolutional neural network optimizer for photosynthetic pigments prediction on plant digital image," Proceedings of the Symposium on Biomathematics, Depok, Indonesia, August 31 - September 1, 2018, pp. 020020-1 - 020020-8. https://doi.org/10.1063/1.5094284.

[31] T.D. Truong, V.T. Nguyen, M.T. Tran, "Lightweight deep convolutional network for tiny object recognition," Proceedings of the 7th International Conference on Pattern Recognition Applications and Methods, Funchal, Portugal, January 16-18, 2018. pp. 675-682. https://doi.org/10.5220/0006752006750682.

[32] A.A. Gitelson, M.N. Merzlyak, Non-destructive assessment of chlorophyll, carotenoid and anthocyanin content in higher plant leaves: principles and algorithm, in: S. Stamatiadis, J.M. Lynch, J.S Schepers (Eds.), Remote Sensing for Agriculture and the Environment. Greece, Ella, 2004, pp. 78-94.

[33] W.D. Huang, K.H. Lin, M.H. Hsu, M.Y. Huang, Z.W. Yang, P.Y. Chao, C.M. Yang, "Eliminating interference by anthocyanin in chlorophyll estimation of sweet potato (Ipomoea batatas L.) leaves," Botanical Study, vol. 55, issue 11, pp. 1-10, 2014 https://doi.org/10.1186/1999-3110-55-11.

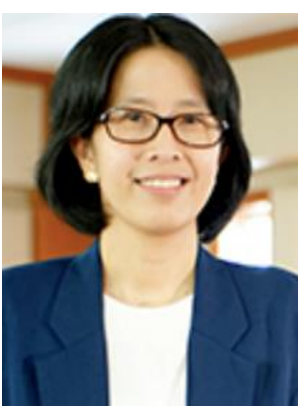

precision agriculture.
KESTRILIA REGA PRILIANTI obtained her undergraduate degree in Statistics from Institut Teknologi Sepuluh Nopember and a master degree in Computer Science from Institut Pertanian Bogor, Indonesia. She has completed her doctoral degree at the Mathematics Department of Universitas Brawijaya, Indonesia. At present, she is also a senior lecturer at the Department of Informatics Universitas Ma Chung, Indonesia. Her current area of research interest is the development of intelligent systems for 


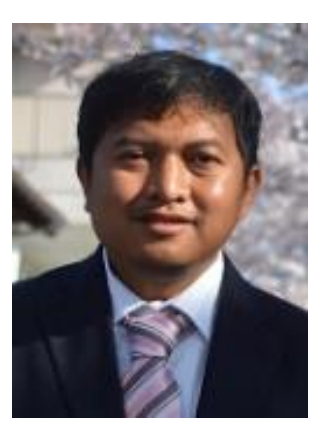

SYIFUL ANAM has taken his bachelor degree in Mathematics from Universitas Brawijaya, Indonesia in 2001 and a master degree in Multimedia Intelligent Network, Electrical Engineering from Institut Teknologi Sepuluh Nopember, Indonesia in 2006. He has completed in a doctoral degree in Information and System Science, Natural Science and Mathematics from Yamaguchi University, Japan in 2015. Since 2002, he is also a lecturer at the Department of Mathematics, Universitas Brawijaya, Indonesia. His current area of research interest is in digital image processing and computational intelligence.

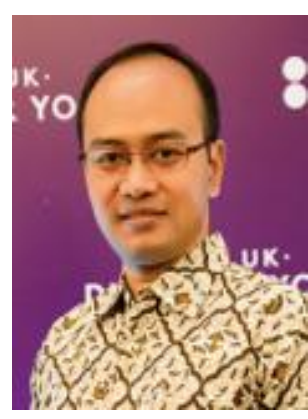

TATAS

HARDO

PANINTINGJATI BROTOSUDARMO is the principal investigator at National COE Ma Chung Research Center for Photosynthetic Pigments (MRCPP) at Universitas Ma Chung, Malang, Indonesia. His research group focus on the analysis plant and microbial photosynthetic pigments using mass spectrometry and optical spectroscopy. He graduated with his diploma degree in Chemistry from Ludwig Maximilian University of Munich, Germany and Ph.D from Glasgow
University, UK with a scholarship from Marie Curie Research Training Network. Currently, he is a fellow of the Indonesian Young Academy of Sciences (ALMI), and a fellow of the Alexander von Humboldt at the Department of Experimental Physics, University of Bayreuth, Germany working on single-molecule imaging spectroscopy.

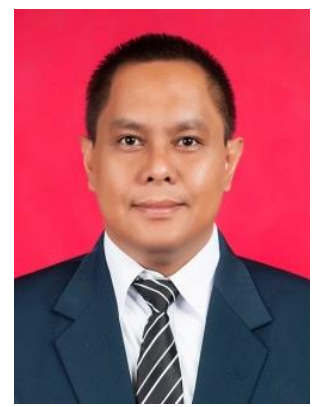

Agus Suryanto received his BSc degree in Mathematics from Universitas Brawijaya, Indonesia, in $1992 . \mathrm{He}$ received his MSc and Ph.D degrees in Applied Mathematics from the University of Twente, the Netherlands, in 1999 and 2003, respectively. He is currently a professor in Applied Mathematics at the Department of Mathematics, Universitas Brawijaya, Indonesia. His main fields of interest are mathematical modeling and numerical computation. 